\title{
L'IMPACT ORGANISATIONNEL DU BIG DATA : CAS DES ENTREPRISES CONGOLAISES
}

\author{
Author(s) / Auteur(s) : \\ Alphonse - Christian IVINZA LEPAPA \\ Docteur en Sciences de Gestion (ULB, Solvay, 2007) \\ DES en Télématique et Organisation (ULB, Faculté des sciences, 1997) \\ Professeur Honoraire de Gestion de la FWB (Belgique). \\ Professeur des Systèmes d'Information de Gestion (MIS) et de Télématique de l'ESU de la RDC. \\ ivinza@hotmail.com
}

\section{Abstract / Résumé :}

Ce travail a pour but d'examiner l'impact organisationnel des Big Data dans le cadre particulier des entreprises d'un pays sous développé (PSD), qu'est la République Démocratique du Congo (RDC). Les recherches sur l'impact organisationnel des Big Data couvrent principalement la structure des ordinateurs, les architectures Big Data (Im Memory, MPP, SSD).

«Les entreprises sont considérées comme des systèmes caractérisés par un besoin fondamental, celui de la survie..» (SEGUIN F. et CHANLAT J.F, 1983, page 12). Cette conception relève du paradigme fonctionnaliste et de l'approche managériale qui met en évidence les éléments majeurs qui doivent être maîtrisés pour assurer la survie d'une organisation : l'adaptation, la réalisation des buts, l'intégration et le maintien des normes.

L'objet du Big Data n'est pas l'information, mais bien la donnée elle-même, valeur de variable manipulée par les TIC. C'est cette démarche que nous souhaitons explorer dans son utilité pour les entreprises Congolaises. L'originalité de Big Data réside en ce qu'elle ne s'appuie pas sur les structurations préexistantes à la collecte de données, mais entend découvrir au sein de ces données des modèles. Par ailleurs, transformer des informations en données induit un coût minima de capture, de transmission et de stockage. Certaines informations sont ainsi transformées en données en raison de leur utilité supposée. Le Big Data est la combinaison d'au moins deux éléments suivants : volume élevé mais peu défini, complexité et technologies. (DELORT, 2015, pages 5 et sq)

L'idée d'un impact organisationnel des TIC et du Big Data semble relever d'un déterminisme technologique renaissant de ses cendres comme à chaque grande vague d'innovations technologiques. (KEFI et KALIKA, 2005, page 45).

Nous examinerons la problématique de l'impact des BIG DATA suivant les aspects suivants :

- Quels outils de modélisation inductive? (Econométrie, Machine Learning, modélisation explicative et prédictive)

- Quelles transformations dans les entreprises?

- Quelles compétences dans quelle structure ? (compétences technologiques, compétences business)

Keywords / Mots-clés : Big Data, information, données, organisation, TIC, modèle, système, causal. 


\section{L'IMPACT ORGANISATIONNEL DU BIG DATA : CAS DES ENTREPRISES CONGOLAISES}

Dans cet article, nous présentons d'abord dans la première section l'objet du Big data, ses origines, sa définition, son historique et le concept de matrice-volume qui explique ce de façon ludique.

Ensuite, nous abordons l'impact organisationnel des TIC et du Big Data en parlant des technologies et architectures Big Data, du traitement de l'information du Big Data, des applications Big Data dans les entreprises, des données de l'internet des objets et de l' 'impact organisationnel du Big Data.

La troisième section dresse un panorama de l'impact des TIC et et du Dig Data dansles PSD et les PVD. A cette occasion on examine les cadres conceptuels des TIC et des SI dans les PSD et PVD,

les études empiriques des TIC et des SI dans les PSD et PVD africains et sub-sahariens et on détermine le ccomportement organisationnel des entreprises des PSD et PVD dans l'adoption du Bige Data.

La quatrième section examine l'impact organisationnel des TIC et Big Data dans les entreprises congolaises. Les points suivant sont abordés : la classification des pays suivant le potentiel de développement informatique (CIDP - Computer Industrial Development Potential), les applications Big Data éligibles dans les entreprises congolaises, l'impact organisationnel du Big Data sur les structures organisationnelles et les postes de travail dans les entreprises congolaises.

La dernière section aborde l'interprétation systémique du comprtement organisationnel d'adoption du Big Data dans les entreprises congolaises. On examine l'iinterprétation systémique du comportement organisationnel des entreprises congolaises.

\section{L'objet du Big Data}

\section{Origines et historique du Big Data}

De l'information à la donnée qui en fin de compte conduit à une sorte d'intelligence augmentée qui crée des nouveaux emplois dans la société, l'automobile.

Selon Pierre Delport « c'est de la profusion de données sur de nombreux domaines que résulte le Big Data ou mégadonnées $»^{1}$.

Trois lois fondent notre capacité à pouvoir transformer, stocker et transmettre les données qui constituent au jourd'hui le Big Data.

Loi de Moore: elle prévoit un doublement de la densité d'inscription sur puce de silicium tous les 18 mois, ce qui permet d'approcher la capacité de calcul de la fonction «tranasformer». Cette loi confirme l'importance de la vitesse de traitement de l'information pour la création de données.

Loi de Kryder: elle concerne un doublement de la capacité de stockage sur disque magnétique (densité) tous les $\mathbf{1 3}$ mois. Cette loi met en évidence l'importance de la conservation de données après sa création, c'est la fonction «stocker ou accumuler».

Loi de Nielsen : elle concerne la fonction de «transmettre » et prévoit un doublement de la capacité des réseaux publics tous les $\mathbf{2 1}$ mois.

«L'originalité de l'approche Big Data réside en ce qu'elle ne s'appuie pas sur les structurations péexistantes à la collecte de données, mais entend découvrir au sein de ces données des modèles. $»^{2}$. Mais, la transformation des informations en données induit un coût minima de capture,

\footnotetext{
${ }^{1}$ DELORT Pierre, Le Big Data, PUF, 2015, Paris, 2015, page 4.

2 DELORT Pierre, Le Big Data, PUF, 2015, Paris, 2015, page 9.
} 
de transmission et de stockage. Certaines informations seront ainsi transformées en données en raison de leur éventuelle utilisation.

\section{Essai de définition du Big Data} suivants ${ }^{3}$ :

Selon les acteurs des TIC, le Big Data serait la combinaison d'au moins deux éléments

$>\quad$ volume élevé mais peu défini,

$>\quad$ complexité et technologies.

Le Big Data «consiste à créer en exploratoire et par induction sur des masses de données à faible densité en information des modèles à capacité prédictive. Les résultats de ces modèles à capacité prédictive puevent être exploités soit de manière autonome, soit par décision. » (p.42).

Le Big Data est différent de de dataminig ( ou fouille de données) du point de vue conceptuel. Le data warehouse où dans lequel s'effectue la fouille de données se base sur un modèle, alors que le Big Data s'efforce de créer des modèles.

\section{Volume et densité des données}

Une BDD est «composée de tables, constituées de lignes et colonnes dont l'intersection forme une cellule.

Le volume de données correspond au nombre de cellules.

La densité des données établit la proportion au sein des cellules, des cellules signifiantes, c'est-àdire non vides ou non nulles ${ }^{4}$.

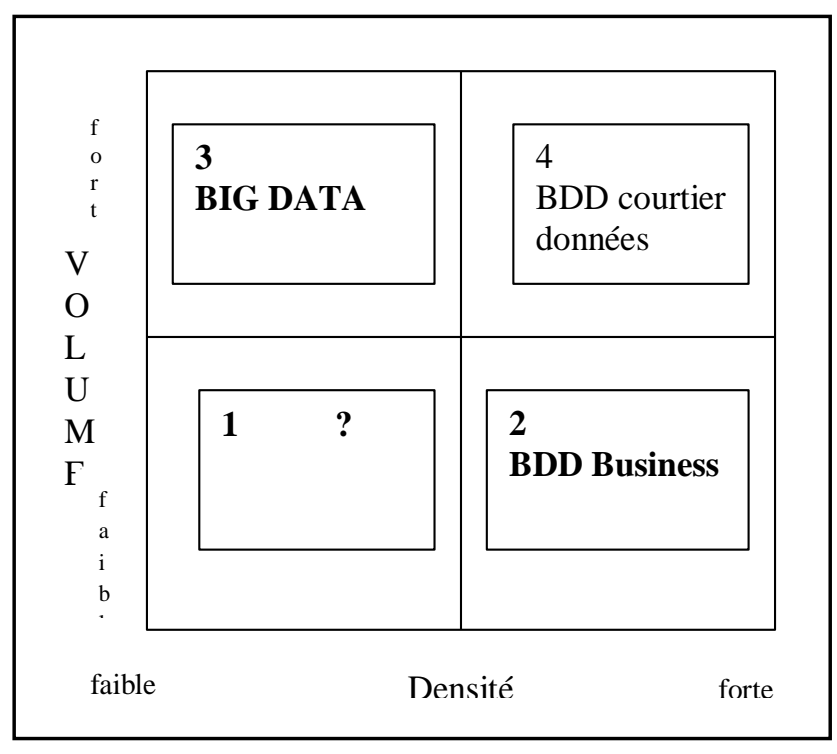

Figure nr. 1 Matrice Volume X Densité

La matrice volume-densité a quatre cadrans qui peuvent être interpretés comme suit :

- Le cadran 1 représente les données de faible densité et de faible volume. Actuellement, il est considéré comme inderteminé, l'aveir dira à qoui il ressemblera.

- Le cadran 2 représente les $\boldsymbol{B D D}$ business, il s'agit des systèmes d'information classique tel que nous les utilisons dans le traitement des informations des entreprises. Dans ce cadran on retrouve les données de forte densité et de faible volume.

- Le cadran 3 représente les données de forte densité et de fort volume. Ce sont les données typiques du Big Data.

\footnotetext{
${ }^{3}$ DELORT Pierre, Le Big Data, PUF, 2015, Paris, 2015, page 6.

${ }^{4}$ DELORT Pierre, Le Big Data, PUF, 2015, Paris, 2015, page 43.
} 
- Le cadran 4 représente les données de faible densité et de fort volume. Ce sont les BDD des courtiers de données qui sont les entreprises qui vendent des informations aux opérateurs économiques leur permettant de prendre certaines décisions.

\section{L'impact organisationnel des TIC et du Big Data}

Les premières automatisations des entreprises qui concernent l'informatique quantitative étaient basées sur le «cost saving » : l'économie des coûts. Plusieurs démarches ont été proposées à ce propos. La plus célèbre est l'analyse des coûts/bénéfices «qui avait pour but d'éclairer l'aspect financier de la décision d'informatiser un système d'information». ${ }^{5}$

Les coûts concernent les frais d'installation et de fonctionnement des matériels, ou de développement des logiciels informatiques. Tandis que les bénéfices peuvent être divisés en avantages tangibles c'est à dire chiffrable monétairement, et les avantages intangibles et donc impondérables et non chiffrables.

Cette philosophie est très vite rattrapée vers les années 1990 avec l'apparition du MIS qui introduit l'aspect organisation préalable à toute informatisation.

\section{Technologies et architectures Big Data}

Les architectures Big Data oscillent autour de trois types : In-Memory, Massivement Parallèle (MPP) et les SSD.

In-Memory permet grâce à une architecture à 64 bits de placer dans une mémoire vive en plus du système d'exploitation et des programmes d'applications un grand volume de données à traiter. Ceci n'était pas possible dans les processeurs 16 et 32 bits.

Le In-Memory provient donc de l'évolution des architectures des processeurs. Pour en bénéficier pleinement, les programmes doivent âtre mis à jour, ce qui permettra une plus grande performance dans la fouille de données.

Massivement Parallèle (MPP) a été conçu pour envoyer les données aux unités de traitement. Le logiciel de référence pour pour ce est le MapReduce de Google.

«Les SSD sont des unités de stockage uniquement constituées de mémoire flash. ». (Delport, p.60). Comparativement au In-Memory, les unités SSD sont : plus lentes, non valatiles, facile à mettre en place, économiques, fiables et totalement extensibles.

\section{Traitement de l'information du Big Data}

Plusieurs traitements sont utilisés dans le Big Data : l'économétrie, les statistiques inductives, les Machines Learning, modélisation explicative et prédictive. Quelle que soit la technique et méthode utilisée pour le traitement de l'information du Big Data tous convergent vers l'usage des statistiques inductives.

Les informaticiens s'intéressent plus aux Machines Learning. Ces machines permettent «d'obtenir des modèles exécutables par les TI à partir des données. » les Big Data utilisent les modèles en induction pour obtenir un programme objectif de prévision. » (Delport p.77).

Quelle est la différence entre Machine Learning et le traitement informatique classique ?

Dans une programmation classique, les données sont traitées par un programme qui produit des résultats pour automatiser les procédures de base ou pour satisfaire les besoins d'information dans l'entreprise. Il s'agit suivant la typologie de Wiseman des systèmes d'information opérationnels ou des systèmes d'information d'aide au management.

\footnotetext{
${ }^{5}$ PEAUCELLE Jean Louis, Informatique pour gestionnaire, Vuibert Gestion, Paris, 1986, page 372.
} 


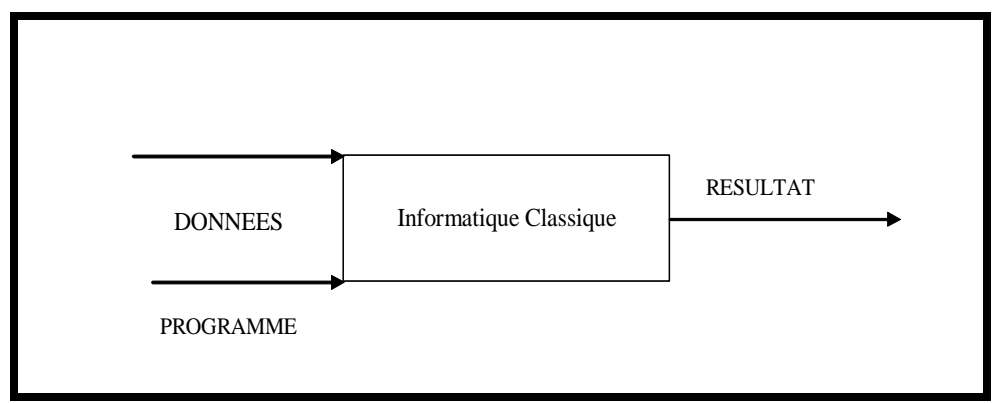

Figure $n$ r. 2. Tritement Informatique classique 6

Tandis que le machine learning (ML) essaye de créer des modèles à partir de données. Les modèles sont tirés par induction pour créer des programmes de prévision. (Delport p. 77) Ce genre de traitement entre dans la catégorie des systèmes d'information stratégique de type $\boldsymbol{S}_{-} \boldsymbol{I S}^{\mathbf{7}}$ : c'est à dire un système qui manipule de l'information stratégique pour construire et suivre une stratégie. ${ }^{8}$

Le système d'information stratégique utilisant des extractions et des analyses par automatisation des besoins d'information, selon Hubert Tardieu et Bernard Guthmann repris par Pateyron est le S-IS. Exemple : l'adaptation du système d'information la volatilité des résultats. ${ }^{9}$

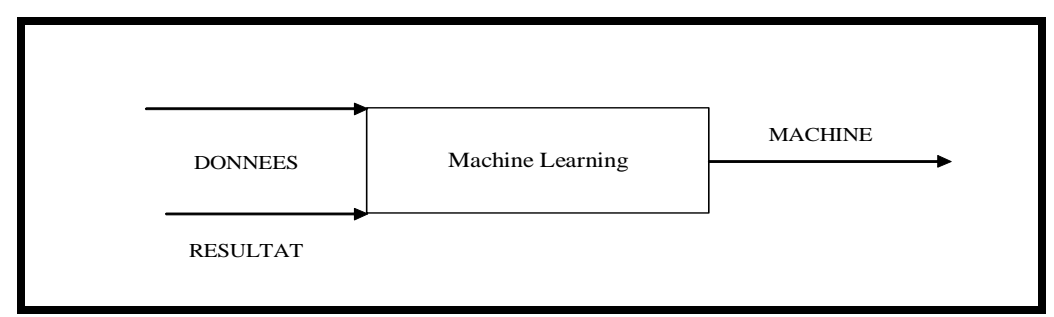

Figure $n r .3$ Machine Learning ${ }^{10}$

Quelles sont les formes principales que peuvent prendre une machine Learning ?

On parle souvent de trois types (Delport, p.78) :

- Dans $M L$ supervisé, en face des données sont fournis les résultats recherchés. Le but recherché est de trouver le modèle qui généralise les relations données résultats.

- Dans ML non supervisé, on recherche les signaux dans les données.

- Pour le ML renforcé, le système interagit avec un environnement changeant.

Nous pouvons dire que le traitement informatique Big Data utilise une démarche différente de la tradition scientifique de la justification des hypothèses basée sur l'expérimentation. La tradition scientifique part d'une série d'hypothèses et essaye de trouver les données et des échantillons pour justifier et conforter les différentes propositions avancées. Ce qu'on appelle hypothesis driven.

La démarche Big Data procède différemment, ce qu'on appelle data driven. Cette démarche essaye à partir des données à susciter des hypothèses.

\section{Quelles sont les applications Big Data dans les entreprises?}

Plusieurs applications concernent le Big Data :

${ }^{6}$ DELORT Pierre, Le Big Data, PUF, 2015, Paris, 2015, page 77.

${ }^{7}$ IVINZA LEPAPA Alphonse, Informatique Stratégique: dre théorique et Applications dans les entreprises Congolaises, page 203, Bookelis, Paris, 2016.

${ }^{8}$ PATEYRON Emmanuel et SALMON Robert, Les nouvelles technologies de l'information et l'entreprise, Economica Gestion Poche, Paris, 1996, page 32.

${ }^{9}$ DUPUY Y. et alii, op.cit., 1989, page 250 et sq ; LESCA Humbert, Système d'information pour le management stratégique de l'entreprise «L'entreprise intelligente», McGrawHill, Paris, 1986, pages 112-122.

${ }^{10}$ DELORT Pierre, Le Big Data, PUF, 2015, Paris, 2015, page 78. 
$>$ les séquenceurs d'ADN,

$>$ les données dactylographiques,

$>$ les données de l'internet des objets.

Pour les pays en développement, nous examinerons seulement l'internet des objets.

\section{Données de l'internet des objets}

L'internet des objets "est constitué de l'ensemble des objets de la vie de tous les jours qui sont lisibles, reco,nnaissables, localisables, adressables et/ou contrôlables par Internet, par les technologies RFID (radio frenquency identifcation), LAN (réseau local), sans fil, WAN (réseau à grande distance). » (p16).

On distingue deux types d'internet à objets :

$>$ les communications d'objet à personne ;

$>$ les communications d'objet à objet interagissant sans intermédiaire humain. (p.16)

Selon Stanford research Instute, l'internet des objets est une innovation technologique destinée à devenir dominante d'ici 2025, elle évolue en quatre étapes (technologie de rupture, p .17) :

$>$ puces RFID-1(utilisation privilégié en logistique pour faciliter les expéditions, les inventaires et prévenir le vol : depuis 2005);

$>$ puces RFID-2 (extension de l'usage aux domaines de la santé, de la surveillance, etc) ;

$>$ loT 1 (géolocalisation permanente, à l'extérieure et à l'intérieur, pour un suivi permanent des personnes et des objets : 2020) ;

$>$ loT 2 (téléopération et téléprésence, surveillance et maîtrise à distance : 2025).

\section{L'impact organisationnel du Big Data}

L'analyse d'impact organisationnel du Big Data de cet article est consacrée aux applications et à l'étude des relations pouvant exister entre les mutations des TIC sur la structure organisationnelle et les postes de travail.

L'avènement du Big Data comme tous les TIC exigent des nouvelles compétences en statistiques, en management et en informatique, qui engendrent des nouveaux postes de travail :

$>$ Chief Data Officer (CDO),

$>$ Data Officier (Analyste),

$>$ Data Protection Officer. compétences.

Ces noveaux postes exigent à divers niveaux une certaine répartition de ces trois

Le CDO est le chef d'orchestre du traitement de données, il est aidé par une équipe d'analystes qui allie les compétences statistiques aux compétences informatiques. Les tâches du DPO sont plus orientées vers les aspects juridiques de la protection des données et de la vie privée.

\section{L'impact organisationnel des TIC et du Big Data dans les entreprises des PSD et PVD}

Dans cette section, nous présentons d'abord l'article de G.B. Davis (1992), qui serait le précurseur des cadres conceptuels des TI et des SI dans la littérature du MIS pour les PSD et les PVD. Ensuite nous survolons, quelques rares études empiriques des TI dans les PSD et PVD, surtout africains et sub-sahariens.

\section{Cadres conceptuels des TIC et des SI dans les PSD et PVD}

Les modèles décrivant les cadres de référence d'adoption et de diffusion des TI et des SI sont rares dans les pays en développement.

Dans son cadre théorique, Gordon B. Davis propose aux pays sous développés et en développement l'utilisation du modèle de Nolan qui, selon lui, a fait ses preuves dans les pays 
développés. On devra alors adapter pour les PSD et PVD, les concepts relatifs à l'accès au capital, à l'infrastructure technologique et à l'adaptation de la culture informationnelle.

Ce cadre porte sur quatre axes ${ }^{11}$ :

$>$ les préalables de l'utilisation des TI et des SI dans les pays sous développés,

$>$ les étapes du modèle de Nolan adapté aux pays sous développés,

$>$ la justification des dépenses informatiques dans les pays sous développés,

$>$ les conditions et les limites pour l'adoption et la diffusion des TI et SI dans les pays sous développés.

$1^{\circ}$ Les préalables de l'utilisation des TI et des SI dans les pays sous développés,

Ce cadre propose cinq impératifs ou préalables de l'utilisation des TI et des SI dans les pays sous développés.

$>$ L'accès au marché des capitaux : la plupart des entreprises des PSD sont des sociétés familiales dont l'accès au capital est difficile. La plupart de travaux sont effectués manuellement, étant donné que l'acquisition des TIC exige la mise en œuvre des sommes importantes.

> Les PSD sont caractérisés par une pénurie des biens et services qu'ils doivent, en général, commander dans les pays occidentaux. Cet impératif est celui de la réduction de la distance des marchés.

> L'acquisition des TI suppose, en général, une infrastructure technique et commerciale solide (réseau téléphonique, compétence managériale, etc.). Cet impératif est aussi repris par l'étude empirique de Boer et Walbeek (1999).

$>$ La culture des PSD est basée principalement sur la tradition orale : l'acquisition des TIC et des SI suppose un changement de mentalité dans le langage de communication.

$>$ La disposition au changement de mentalité est aussi un impératif important, selon Davis, pour passer d'une tradition orale à une tradition écrite. Cet impératif est discutable et contrarié par la génération Internet, laquelle devrait être universelle quelle soit le pays. ${ }^{12}$

$\underline{2^{\circ} \text { Les étapes du modèle de Nolan adapté aux pays sous développés }}$

GB Davis considère que les quatre étapes originelles du modèle de Nolan peuvent être appliquées aux PSD. Mais elles doivent être couplées d'un apprentissage organisationnel spécifique à chaque étape. C'est ainsi qu'il propose qu'à l'étape Initiation que les premiers contacts avec l'informatique se fassent avec un micro-ordinateur.

$\underline{3^{\circ} \mathrm{La} \text { justification des dépenses informatiques dans les pays sous développés, }}$

L'acquisition des TIC dans les organisations provient, en général, de deux types de motivations : économiques et politiques.

$\underline{4^{\circ} L \text { es conditions et les limites pour l'adoption et la diffusion des TIC et SI dans les pays sous développés. }}$ Un certain nombre des conditions limite l'adoption et la diffusion des TIC et des SI dans les PSD et PVD. Parmi ces conditions :

$>$ Dans le système éducatif, l'informatique n'est pas intégrée dans certains établissements de ces pays dans l'enseignement primaire ni secondaire. Cette condition est discutable et toute somme dépassée.

$>$ Il existe des barrières culturelles à l'utilisation des ordinateurs.

$>$ Il existe une tendance généralisée à ignorer des considérations managériales et organisationnelles dans les entreprises de ces pays.

\footnotetext{
${ }^{11}$ DAVIS Gordon B., (1992) A Model for Adoption and Diffusion of Information Systems in Less Developed Countries, in PALVIA Shailendra C. Jain , PALVIA Prashant C. and ZIGLI Ronald M., (1992) The Global Issues of Information Technology Management, Idea Group Publishing, Harrisburg.

12 JACOB Réal, Génération Internet, in Revue internationale de Gestion, volume 27, numéro 2, Eté 2002, page 46.

PARE Guy, La génération Internet : un nouveau profil d'employés, in Revue internationale de Gestion, volume 27, numéro 2, Eté 2002, pages 47-53.
} 
$>$ Le non respect des propriétés intellectuelles occasionne le piratage des logiciels et des ouvrages par des copies illégales. Le besoin d'apprendre et la pauvreté justifient ce comportement.

\section{Etudes empiriques des TIC et des SI dans les PSD et PVD africains et sub-sahariens}

Nous avons recensé quelques travaux empiriques parmi lesquels :

$>$ Le modèle et les conditions pour l'usage des systèmes d'information stratégiques dans les pays en développement (Palvia, Palvia et Zigli ; 1992) ${ }^{13}$

$>$ Le développement des systèmes d'information dans les pays en développement (Ming-Te LU et Farell, 1990), ${ }^{14}$

$>$ Les tendances émergentes des TIC : implication pour les pays en développement (Bowonder, Miyqake, Singh ; 1993). ${ }^{15}$

Qu'à cela ne tienne, ces travaux reflètent les idées contenues dans le modèle d'adoption des TIC et des SI présenté par G. B. Davis (1992)

$1^{\circ}$ Les recherches dans les pays africains sub - sahariens

Les recherches empiriques d'impact des TIC sont rares dans les pays sous développés et plus précisément en Afrique Sub- Saharienne.

Palvia, Palvia et Withworth (2002, p.411) font référence à quelques recherches dans les pays africains en développement comme le Nigéria, le Kenya et le Zimbabwe; mais non dans les pays africains sous développés.

$\underline{2^{\circ} \text { Guide pour la formulation des politiques des TIC dans les pays en développement }}$

Dans une recherche commanditée par le gouvernement hollandais, De Boer et Walbeek (1999) ont étudié les possibilités de solutionner les écarts constituant les obstacles au développement de la télématique dans les pays en développement.

Suivant les résultats de cette étude les obstacles majeurs proviennent, d'une part, des facteurs de régulation et d'infrastructure; et d'autre part, de l'inadéquation de l'offre des providers et de la demande des utilisateurs des pays du sud. Pour combler ces insuffisances le gouvernement, les PTT, les industries et les organisations internationales doivent supporter les activités de développement. ${ }^{16}$

$\underline{3^{\circ} \text { Les caractéristiques d'adoption et de diffusion des TIC et des SI dans les pays sous développés }}$

Une des rares études effectuées dans les pays africains en développement est celle de Zigli (1990) au Kenya et au Zimbabwe. ${ }^{17}$ Les deux pays sont considérés selon la classification des Nations Unies comme des pays ayant des potentiels informatiques essentiels (basic).

Selon Zigli, les sept caractéristiques principales relatives à l'adoption et à la diffusion des TI et des SI pour cette catégorie des pays sont :

$>$ obsolescence des équipements informatiques (hardware),

$>$ obsolescence des applications informatiques (software d'applications),

$>$ prolifération des commerçants génériques des logiciels et matériels informatiques (proliferation of mixed vendor shop hardware and software),

$>$ disponibilité limitée des compétences informatiques et de la formation du personnel,

${ }^{13}$ PALVIA Prashant, PALVIA Shailendra and ZIGLI Ronald M., Models and requirements for using Strategic Information systems in Developing nations, in International Journal of Information Management, volume 10, issue 2, June 1990, pages $117-126$

${ }^{14}$ LU Ming-TE and FARRELL Crumpton, Information Systems development in developing countries : An evaluation and recommendations, in International Journal of Information Management, volume 10, issue 4, December 1990, pages 288296.

${ }^{15}$ BOWONDER B., MIYQAKE T. and SINGH T. Monish, Emerging trends in information technology: Implications for developing countries, in International Journal of Information Management, volume 13, issue, June 1993, pages 183-204.

${ }^{16}$ DE BOER Sirp J. and WALBEEK Mirjam M., Information Technology in developing countries : A studies to guide policy formulation, in International Journal of Information Management, volume 19, 1999, pages 207-218.

17 ZIGLI, R.M., Rank order of African MIS issues based on personal interviews with selected Information systems executives, Working paper, The Citadel, 1990, Charleston. 
$>$ interventionnisme gouvernemental possible dans l'acquisition des TIC,

$>$ difficulté d'établir des standards professionnels pour les TIC,

$>$ la productivité de systèmes d'information doit être améliorée.

$\underline{4^{\circ} \text { Les recherches en Afrique du Sud }}$

L'Afrique du Sud est un pays en développement, on y observe la naissance d'une littérature active du MIS. Déjà en 1998, Adeline Du Toit note l'utilisation des TIC dans les entreprises manufacturières pour gagner des avantages compétitifs. ${ }^{18}$

Une étude de l'OCDE (2002) mentionne aussi la présence d'une littérature d'impact des TIC en Afrique du Sud, plus précisément, sur les perspectives et défis de l'E-business dans le secteur sudafricain des pièces automobiles (Moodley, 2002, p.75) ${ }^{19}$ et le commerce électronique (Jackson et Eksteen, 2002, p.201). ${ }^{20}$

$5^{\circ}$ Problématique d'adoption et de diffusion des TIC en Afrique de l'Ouest et spécialement au Nigéria.

Janczewski Lech J. (1992) dans une étude empirique a analysé les facteurs d'implantation des TI dans les pays sous développés. ${ }^{21} \mathrm{Il}$ a pris dans son échantillon les pays de l'Afrique de l'Ouest. Cet échantillon comprend le Nigéria, le Sénégal, la Gambie, la Guinée, le Mali, la Côte d'Ivoire, le Burkina Faso, le Ghana, le Bénin, le Niger et le Cameroun. Le Nigéria est le pays le plus étendu et le plus peuplé de la région, cette analyse porte essentiellement sur sa problématique.

\section{Comportement organisationnel des entreprises des PSD et PVD dans l'adoption du Big Data}

L'examen de la littérature du MIS nous permet d'anticiper le comportement des entreprises de certains pays en développement face à l'adoption des nouvelles technologies, comme le Big Data.

Certains pays africains et plus précisement, les pays anglo-saxons ont une grande propension à adapter les TIC et donc le Big Data.

L'Afrique du Sud est le pays le plus informatisé selon la littérature suivi par les autres pays anglophones le Kenya, le Zimbabwe et le Nigéria. Tandis que les autres pays surtout francophones semblent assez timide dans l'adoption des TIC, il s'agit du Sénégal, de la Gambie, de la Guinée, du Mali, de la Côte d'Ivoire, du Burkina Faso, du Bénin, du Niger et du Cameroun.

Janczewski Lech J. (1992) qui a travaillé pendant trois ans dans un projet d'informatisation de ces pays a relevé trois types de problème d'implantation des TI : des problèmes techniques, des problèmes économiques et des problèmes culturels.

\section{L'impact organisationnel du Big Data dans les entreprises congolaises}

Classification des pays suivant le potentiel de développement informatique (CIDP - Computer Industrial Development Potential)

Nous référant à la classification du niveau de développement économique des pays utilisée par Palvia et alii qui ont analysé les différents thèmes de recherche d'impact des TI par régions et pays, nous pouvons considérer la RDC comme un pays en de développement ou sous développé.

Ce que confirme d'ailleurs Del Castillo dans une étude de l'OCDE (2003, p.1). Cette étude classe le développement économique des pays en quatre catégories :

\footnotetext{
${ }^{18} \mathrm{Du}$ TOIT Adeline, Information Management in south African manufacturing enterprises, in International journal of Information Management, volume 18, issue 3, 1998, pages 205-213.

19 MOODLEY Sagren, Perspectives de l'E- business pour le secteur sud-africain des pièces automobiles page 75 in GOLDSTEIN Andrea et O'CONNOR David (éditeur), Commerce électronique et Développement, Etudes du centre de développement, OCDE, 2002.

${ }^{20}$ JACKSON Carey-Ann et EKSTEEN Johan, Création de contenu local et commerce électronique : point de vue sudafricain page 201 in GOLDSTEIN Andrea et O'CONNOR David (éditeur), Commerce électronique et Développement, Etudes du centre de développement, OCDE, 2002.

${ }^{21}$ JANCZEWSKI LECH J, Factors of information Technology Implantation in Under-Developed countries : Example of the West Africa Nations, 1992, page 187-212 in PALVIA, PALVIA and ZIGLI, op. cit., 1992.
} 
$>$ pays développé,

$>$ pays nouvellement industrialisé,

$>$ pays en développement

$>$ et pays sous développé (Palvia et alii. 2002 p.403).

Cette classification est inspirée par celle des Nations Unies (Porat, 1977 ; Kalman, 1979) qui classe les pays suivant leur potentiel de développement informatique (CIDP - Computer Industrial Development Potential) :

$>$ pays à potentiel informatique avancé,

$>$ pays à potentiel informatique opérationnel,

$>$ pays à potentiel informatique essentiel

$>$ et pays à potentiel informatique embryonnaire.

La République Démocratique du Congo peut être considéré comme étant un pays à potentiel informatique essentiel et embryonnaire.

\section{Quelles sont les applications Big Data éligibles dans les entreprises congolaises?}

Plusieurs applications concernent le Big Data :

$>$ les séquenceurs d'ADN,

$>$ les données dactylographiques,

$>$ les données de l'internet des objets.

$\mathrm{Vu}$ le niveau élémentaire d'informatisation (essentiel et embryonnaire) des entreprises congolaises et l'étendu du pays ; parmi les applications Big Data ci-dessus. Les entreprises choisiront de traiter les données dactylographiques et l'attrait universel de la télématique conduise aux données de l'internet des objets.

\section{$1^{\circ}$ Difficultés d'adoption du Big Data}

Suivant le cadre conceptuel de GB DAVIS, l'une des raisons à la base serait l'accès au marché des capitaux. La plupart des entreprises des PSD sont des sociétés familiales dont l'accès au capital est difficile. La plupart de travaux sont effectués manuellement, étant donné que l'acquisition des TIC exige la mise en œuvre des sommes importantes.

Les autres raisons seraient économiques et politiques.

Les raisons économiques sont basées sur la performance, les économies des dépenses de main d'œuvre ou de traitement de l'information et l'acquisition des avantages compétitifs. Les dépenses d'acquisition font défi aux économies des coûts de main d'œuvre obtenu par les TIC. Cela étant donné que dans les PSD, la main d'œuvre est d'habitude moins chère.

Souvent ce sont les raisons politiques telles que donner une image de marque à l'entreprise qui pèseront plus pour l'acquisition de l'informatique que la rationalisation des méthodes de gestion. Ce n'est qu'après l'acquisition qu'on pense parfois aux performances, c'est alors que le critère d'apprentissage organisationnel doit être pris en compte.

\section{Impact organisationnel du Big Data sur les structures organisationnelles et les postes de travail dans les entreprises congolaises}

Les entreprises congolaises ont un obstacle (catharsis) mental qui les empêche d'adopter des nouvelles TIC comme le Big Data. Dans le cadre conceptuel de Gordon Davis, il s'agit existe d'une tendance généralisée à ignorer des considérations managériales et organisationnelles dans les entreprises congolaises. Cette condition est vérifiée quand on observe les entreprises d'Etat d'un pays comme la $\mathrm{RDC}^{22}$.

\footnotetext{
${ }^{22}$ IVINZA LEPAPA A.C., Informatique Stratégique : Cadre théorique et Applications dans les entreprises congolaises, Bookelis, 2016, Paris, page 218.
} 
De même, il existe des barrières culturelles à l'utilisation des ordinateurs. Cette condition est contrariée par l'étude de l'avènement de la génération Internet ${ }^{23}$.

$1^{\circ}$ Positionnement des fonctions Big Data

Quel que soit les obstacles mentaux et culturels des entreprises congolaises, des problèmes incontournables persistent et doivent être résolus parmi lesquels: où travaillerons les nouveaux métiers générés par cette technologie?

Il s'agit du Chief Data Officer (CDO), du Data Officier (Analyste) et du Data Protection Officer.

Ces nouveaux métiers doivent ils être dans une structure autonome où doivent ils être intégrés dans la Direction des Systèmes Informatiques pour bénéficier de la synergie de cette fonction comprenant l'infrastructure matérielle et les compétences informatiques? Cela dépend de la position du centre informatique et de sa taille qui dépend de l'importance accordée par la Direction Générale au traitement de l'information.

La place de l'informatique dans l'organigramme d'une entreprise ou la position hiérarchique d'un centre informatique est un sujet très préoccupant, alors que le traitement de l'information et les ordinateurs sont dans un développement exponentiel. Il y a lieu d'intégrer les nouveaux métiers dans le positionnement de la Direction des systèmes Informatiques. Mais il faut montrer la nécessité de ce rattachement et de s'assurer si l'organisation sociale le facilite.

Puisque la position hiérarchique d'un service ne peut être isolée, il faut situer l'informatique dans une structure organisationnelle globale de l'entreprise. Il existe plusieurs types de structure dans une entreprise. Elles peuvent être matérielles, sociales, économiques, etc.

Dans cet article, seule la structure sociale ou humaine, celle qui étudie les relations entre les hommes dans une organisation de travail nous intéresse. A ce point de vue, nous distinguons la structure sociale informelle de la structure sociale formelle. Nous nous limiterons à la structure sociale formelle de l'entreprise. Il s'agit des relations entre agents d'une société, relations qui sont explicitement établies par la Direction. Elles figurent dans les organigrammes et sont détaillées dans les descriptions des divers postes de travail. Malheureusement cette structure ne reflète pas toujours la réalité telle qu'elle devrait être vécue dans l'entreprise.

\section{Interprétation systémique du comportement organisationnel d'adoption du Big Data dans les entreprises congolaises}

\section{Interprétation systémique du comportement organisationnel des entreprises congolaises}

La théorie des systèmes « est la base sur laquelle s'appuient les théoriciens de la contingence, ainsi que sur une vision structuro- fonctionnaliste suivant laquelle la survie des organisations dépend de leur adaptation à l'environnement $\gg .^{24}$

$\mathrm{Du}$ point de vue systémique, nous commenterons quatre propriétés pour expliquer le comportement organisationnel d'adoption du Big Data dans les entreprises congolaises : réversibilité et irréversibilité, convergence et divergence, auto - apprentissage, auto- organisation.

\section{$1^{\circ}$ Réversibilité et Irréversibilité}

Face aux événements transitoires, l'adoption du Big Data sera réversible et face aux événements définitifs, ce modèle sera irréversible.

Dans l'adoption des TIC, il existe un délai entre le système des causes et celui des effets. Les TIC apparaissent avant la formulation des stratégies et la conception des systèmes d'information ainsi que des structures organisationnelles (impératif technologique).

\footnotetext{
23 IVINZA LEPAPA A.C., Informatique Stratégique: Cadre théorique et Applications dans les entreprises congolaises,Bookelis, 2016, Paris, page 219.

24 GUITTEREZ Luiz Homero, thèse citée, 1993, page 47.
} 
Les TI ont des transformations continues et engendrent des évènements définitifs irréversibles. Dans les entreprises congolaises, on a constaté qu'à l'apparition des nouveaux types de matériels informatiques, l'échange par document diminue au profit des médias plus améliorés.

\section{$2^{\circ}$ Convergence et Divergence}

«Bien entendu, un même système peut connaître des phases successives de convergence et de divergence. En particulier, il se forme généralement dans une phase de convergence et se détruit dans une phase de divergence». ${ }^{25}$

Dans l'adoption des TIC certaines entreprises commencent par une phase de convergence pour la plupart des entreprises d'Etat avec des hiérarchies électroniques conduisant à la mise en place des organisations réseaux LAN Intranet. La phase de divergence est présente et se caractérise par une désynchronisation progressive qui amène à la fermeture des agences de l'intérieur face à la concurrence du secteur. C'est le cas des entreprises privées.

\section{$\underline{3^{\circ} \text { Auto -apprentissage }}$}

«L'apprentissage exige en effet que la relation entrée-sortie ne soit ni trop lâche (pour permettre une réponse adaptée au stimulus), ni trop rigide (pour pouvoir modifier cette réponse si elle est inadéquate)». ${ }^{26}$

Le fait que la stratégie des entreprises congolaises subisse des transformations réversibles permet aux managers d'adapter leur comportement face aux convergences et aux divergences. Ces transformations conduisent soit à l'implosion, soit à l'explosion de l'impact des TIC et du Big Data.

\section{$\underline{4^{\circ} \text { Auto- organisation }}$}

«Un système peut ainsi connaître des phases de déstructuration et de restructuration se recouvrant partiellement. (...) L'auto -organisation peut intervenir lors de modifications lentes et continues de l'environnement du système.(...) Mais l'auto -organisation intervient également lors de modifications brutales. $\gg^{27}$

L'adoption des TIC ou du Big Data s'apparente à un système critique auto - organisé qui est faiblement chaotique. Les systèmes critiques auto - organisés «évoluent vers un état critique tel qu'une petite perturbation déclenche une réaction en chaîne qui touche un nombre aléatoire d'éléments du système. Selon le nombre d'éléments touchés, la perturbation provoque un événement mineur ou une catastrophe $\gg .^{28}$

Le cas d'auto -organisation le plus perceptible est celui des entreprises du guichet unique qui ont adopté les SISED et une organisation réseau WAN Extranet sans les avoir prévu. Ces entreprises doivent combler un fossé organisationnel en réalisant d'abord une intégration interne de leurs organisations pour bien asseoir l'intégration externe (Baile, 1997b, p.169).

\section{Comportement organisationnel des entreprises congolaises}

Le comportement organisationnel, qui est le système opérateur «subit les entrées et produit les sorties du système, mais il est guidé par les commandes imposées par le régulateur». ${ }^{29}$

Le système opérateur effectue une transformation qualitative des entrées sorties et fonctionne comme un système causal déterminé (certain) ou aléatoire (stochastique).

Il est certain quand toutes les données d'entrée sont connues, il peut alors permettre une prévision parfaite du comprtement organisationnel.

Il est aléatoire quand les données d'entrée ne sont pas toutes connues et ne peut permettre, dès lors, qu'une prévision imparfaite du comportement organisationnel.

\footnotetext{
${ }^{25}$ WALLISER Bernard, op.cit., Paris, 1977, page 85.

${ }^{26}$ WALLISER Bernard, op.cit., Paris, 1977, page 86.

${ }^{27}$ WALLISER Bernard, op.cit., Paris, 1977, page 87.

${ }^{28}$ TREMBLAY Christian, op.cit., 1992, page 132.

${ }^{29}$ WALLISER Bernard, op.cit., 1977, page 27.
} 
Le modèle de recherche développé par cet article ne vise pas à attacher des nouvelles TIC à un modèle organisationnel de valeur universelle, mais à mettre en évidence les principes organisateurs qui permettront aux entreprises congolaises de faire face à certains changements de l'environnement et des TIC.

Leifer (1988) avait proposé une correspondance entre le CBIS (computer based information system) et les configurations structurelles de Mintzberg, cette correspondance a été très vite déphasée avec l'évolution exponentielle des TIC. ${ }^{30}$ "Un acquis de l'observation et de 1 'analyse des entreprises est qu'aucun modèle d'organisation n'a de valeur universelle. Il semble donc illusoire de vouloir associer aux TIC de nouvelles formes universelles d'organisation. De façon plus modeste, il est question de rechercher quels sont les principes organisateurs qui permettent aux entreprises de faire face aux défis de la fin de ce siècle $»{ }^{31}$

Les principes organisateurs généralement reconnus dans l'impact des TI sont: la contingence, la cohérence, l'intelligence, la vigilance (Pateyron et Salmon, 1996, p.79) et la stratégie (Venkatraman, 1991).

Dans les entreprises congolaises, ces principes sont utilisés de cette façon :

$>$ la contingence des TI sur les structures est établie, mais elle est parfois faible ou inexistante sur les SI,

$>$ la stratégie est utilisée dans les entreprises manifestant le besoin d'utiliser le Big Data,

$>$ l'intelligence, la cohérence et la vigilance ne sont pas utilisées.

Le comportement organisationnel de la moitié des entreprises observées a un rôle opérateur passif, ce qui peut conduire à des systèmes auto critiques organisés faiblement chaotiques pouvant amener un grand dysfonctionnement organisationnel. L'autre moitié des entreprises a un comportement organisationnel qui a un rôle opérateur actif.

\section{Conclusion générale}

L'objet du Big Data n'est pas l'information, mais bien la donnée elle-même, valeur de variable manipulée par les TIC. C'est cette démarche que nous avons exploré dans son utilité pour les entreprises Congolaises.

L'originalité de Big Data réside en ce qu'elle ne s'appuie pas sur les structurations préexistantes à la collecte de données, mais entend découvrir au sein de ces données des modèles. Par ailleurs, transformer des informations en données induit un coût minima de capture, de transmission et de stockage.

Certaines informations sont ainsi transformées en données en raison de leur utilité supposée. Le Big Data est la combinaison d'au moins deux éléments suivants : volume élevé mais peu défini, complexité et technologies.

Nous avons examiné la problématique de l'impact des BIG DATA suivant les aspects suivants : less outils de modélisation inductive (Econométrie, Machine Learning, modélisation explicative et prédictive), les transformations dans les entreprises, et les compétences exigées (compétences technologiques, compétences managériales).

Quelle est la solution à adopter pour l'amélioration du comportement organisationnel des managers congolais?

Le comportement organisationnel de ces entreprises a un rôle opérateur tantôt passif, tantôt actif qui souffre d'une carence de cohérence, de vigilance et de stratégie. Il ne peut être amélioré que par l'acquisition des compétences au moyen d'un apprentissage à quatre niveaux : technologique, d'affaires, stratégique et organisationnelle.

\footnotetext{
${ }^{30}$ LEIFER Richard, Matching Computer-Based Information Systems with Organizational Structures, in MIS Quarterly, Volume 12, nr 1, March 1988, pages 63-73.

31 PATEYRON Emmanuel et SALMON Robert, Les nouvelles technologies de l'information et l'entreprise, Economica Gestion Poche, Paris, 1996, page 79.
} 


\section{RÉFÉRENCES}

- BOWONDER B., MIYQAKE T. and SINGH T. Monish, Emerging trends in information technology: Implications for developing countries, in International Journal of Information Management, volume 13, issue, June 1993, pages 183-204.

- DAVIS Gordon B., (1992) A Model for Adoption and Diffusion of Information Systems in Less Developed Countries, in PALVIA Shailendra C. Jain, PALVIA Prashant C. and ZIGLI Ronald M., (1992) The Global Issues of Information Technology Management, Idea Group Publishing, Harrisburg.

- DE BOER Sirp J. and WALBEEK Mirjam M., Information Technology in developing countries : A studies to guide policy formulation, in International Journal of Information Management, volume 19, 1999, pages 207-218.

- DELORT Pierre, Le Big Data, PUF, 2015, Paris, 2015, page 43.

- DUBE Line et PARE Guy, Les Technologies de l'Information et l'organisation à l'ère du virtuel employés, in Revue internationale de Gestion, volume 24, numéro 2, Eté 1999, pages $14-22$

- DUPUY Y. et alii, op.cit., 1989, page 250 et sq ; LESCA Humbert, Système d'information pour le management stratégique de l'entreprise «L'entreprise intelligente», McGrawHill, Paris, 1986, pages 112-122.

- Du TOIT Adeline, Information Management in south African manufacturing enterprises, in International journal of Information Management, volume 18, issue 3, 1998, pages 205-213.

- JACKSON Carey-Ann et EKSTEEN Johan, Création de contenu local et commerce électronique: point de vue sud-africain page 201 in GOLDSTEIN Andrea et O'CONNOR David (éditeur), Commerce électronique et Développement, Etudes du centre de développement, OCDE, 2002.

- JANCZEWSKI LECH J, Factors of information Technology Implantation in UnderDeveloped countries : Example of the West Africa Nations, 1992, page 187-212 in PALVIA, PALVIA and ZIGLI, op. cit., 1992.

- JACOB Réal, Génération Internet, in Revue internationale de Gestion, volume 27, numéro 2, Eté 2002, page 46.

- IVINZA LEPAPA Alphonse, "Informatique Stratégique : Cadre théorique et Applications dans les entreprises congolaises ", Bookelis, Paris, 2016.

- IVINZA LEPAPA Alphonse, " Analyse de l'introduction de l'EDI dans les entreprises congolaises : une contribution à l'impact organisationnel des TI ", Tome 1, EUE, 2010.

- LEIFER Richard, Matching Computer-Based Information Systems with Organizational Structures, in MIS Quarterly, Volume 12, nr 1, March 1988, pages 63-73.

- LU Ming-TE and FARRELL Crumpton, Information Systems development in developing countries: An evaluation and recommendations, in International Journal of Information Management, volume 10, issue 4, December 1990, pages 288-296.

- MOODLEY Sagren, Perspectives de l'E- business pour le secteur sud-africain des pièces automobiles page 75 in GOLDSTEIN Andrea et O'CONNOR David (éditeur), Commerce électronique et Développement, Etudes du centre de développement, OCDE, 2002.

- OCDE (1991), La dimension économique des normes en matière de technologies de l'information, politiques d'information, d'informatique et de communications $\mathrm{n}^{\circ} 25$, Paris.

- PARE Guy, La Génération Internet : un nouveau profil d'employés, in Revue internationale de Gestion, volume 27, numéro 2, Eté 2002, pages 46-53.

- PALVIA Shailendra C. Jain, PALVIA Prashant C. and ZIGLI Ronald M. (1992), The Global Issues of Information Technology Management, Idea Group Publishing, Harrisburg.

- PALVIA Prashant, PALVIA Shailendra and ZIGLI Ronald M., Models and requirements for using Strategic Information systems in Developing nations, in International Journal of Information Management, volume 10, issue 2, June 1990, pages 117-126. 
- PATEYRON E. (1994), Le management stratégique de l'information, Economica Gestion Poche, Paris.

- PARE Guy, La Génération Internet : un nouveau profil d'employés, in Revue internationale de Gestion, volume 27, numéro 2, Eté 2002, pages 46-53.

- PATEYRON E. et SALMON R. (1996), Les nouvelles technologies de l'information et l'entreprise, Economica Gestion Poche, Paris.

- PEAUCELLE Jean Louis (1986), Informatique pour gestionnaire, Vuibert Gestion, Paris.

- PEAUCELLE Jean-Louis (1999), Systèmes d'information : le point de vue des gestionnaires, Economica Gestion, Paris.

- TREMBLAY Christian (1992), L'informatique du décideur : pour intégrer l'informatique à la stratégie de l'entreprise, Marabout, Paris.

- WALLISER Bernard, Systèmes et Modèles : Introduction critique à l'analyse des systèmes, Editions du Seuil, Paris, 1977.

- WILKIN Luc (1986), Informatique et Organisations, Actes du colloque de Nivelles: L'ordinateur, L'homme et L'organisation $2^{\text {ème }}$ partie, PUB.

- WILKIN Luc, (1999) Principes généraux d'organisation et de gestion, 2ième édition, PUB

- WISEMAN C., (1985) Strategy and computers :Information systems as competitive weapons, Homewood, IL : Dow Jones-Irwin, pour l'édition française L'informatique stratégique : nouvel atout de la compétitivité, Edition d'organisation, Paris, 1987.

- ZIGLI, R.M., Rank order of African MIS issues based on personal interviews with selected Information systems executives, Working paper, The Citadel, 1990, Charleston. 
